\title{
INVASIVE MENINGOCOCCAL DISEASE WITH FATAL OUTCOME IN A SWISS STUdENT VISITING BERLIN
}

\author{
I Zuschneid ${ }^{1}$, A Witschi², L Quaback ${ }^{3}$, W Hellenbrand (hellenbrandw@rki.de) ${ }^{4}$, N Kleinkauf ${ }^{4}$, D Koch ${ }^{5}$, Gérard Krause ${ }^{4}$ \\ 1. Kreuzberg-Friedrichshain Health Department, Berlin, Germany \\ 2. Health Department of Basel-Stadt, Basel, Switzerland \\ 3. Senate Department for Health, Environment and Consumer Protection, Berlin, Germany \\ 4. Department of Infectious Disease Epidemiology, Robert Koch-Institute, Berlin, Germany \\ 5. Department of Communicable Diseases, Federal Department of Home Affairs FDHA, Federal Office of Public Health FOPH, Public \\ Health Directorate, Bern, Switzerland
}

\begin{abstract}
Following the fatal invasive meningococcal disease in a Swiss student who had been visiting Berlin, several public health institutions on local, regional and national level cooperated to ensure that the appropriate measures such as contact tracing and post exposure prophylaxis were taken to prevent further cases. The incidence highlighted the importance of early disease notification and showed that if an infectious disease requiring public health action occurs in an international context, it is vital that relevant information is communicated to all levels of the public health systems of the countries involved.
\end{abstract}

An 18 year-old Swiss student travelled to Berlin on a class trip by night train on 12/13 October 2008, where the group stayed at a hostel. During the night from 17 to 18 October, the student complained of severe headache, back pain and vomiting and presented at a local hospital on 18 October, where invasive meningococcal disease was diagnosed by culture in blood and cerebrospinal fluid. Despite intensive care measures, the patient died on 20 October. The isolate was sent to the German National Reference Centre for Meningococci, where the serogroup was determined as W135.

Contact tracing and measures to prevent further cases

\section{Switzerland}

The patient's classmates returned to Basel, Switzerland by night train on 18 October. Before returning, the travel group informed the Cantonal Health Department in Basel about the situation and chemoprophylaxis was provided to the students immediately upon their arrival. The students were counselled by the child and youth health division of the cantonal health department and a letter was sent out to parents. Close household contacts of the patient, who had stayed in Basel received chemoprophylaxis one day later. In addition, a press release informing about the case and the chemoprophylaxis that had been carried out was issued by the cantonal health department in Basel on 20 October.

\section{Germany}

The hospital in Berlin where the patient was treated notified the case to the local district health department by fax on Sunday, 19 October, which in turn informed the Berlin Senate Department for
Health, Environment and Consumer Protection (state level) and the Robert Koch-Institute (RKI) on 20 October 2008. Furthermore, a message about the incident was posted on the Early Warning and Response System (EWRS) on 21 October by the RKI.

In the course of the ensuing contact tracing, information was repeatedly exchanged between the local health departments in Berlin and Basel on 20 October. The Berlin health departments issued a first press release on 21 October informing about the case. Investigations revealed that a young woman who was not part of the Swiss travel group had travelled to Berlin in the same train compartment as the patient. In addition, the patient had visited a local nightclub on 15 and 17 October, where classmates described close contact with several persons not part of the travel party. The local Berlin health authority therefore issued a second press release later on 21 October informing about symptoms and transmission of meningococcal disease and requesting the young woman who had travelled to Berlin in the same compartment, whose first name appeared in the press release, as well as persons who had had close contact with the patient in the club, to contact the local health department for assessment of the need for chemoprophylaxis.

The investigations revealed that the patient had had breakfast in a common room at the hostel together with travel groups from three German federal states as well as from the Netherlands. Although it was unlikely that close contact with the patient beyond possibly sharing the same table at breakfast had taken place, the supervisors of the travel groups, the respective German state health departments and the national public health institute in the Netherlands were informed about the case on 21 October. The respective federal state or country authorities were provided with information about the travel parties, including contact information for the group supervisors, to enable an assessment of the possible need for chemoprophylaxis and appropriate counselling by the responsible health authorities. A number of individual travellers from Germany and from other European countries also stayed at the hostel. However, as the investigations did not indicate close contact between the patient and these travellers, further contact tracing was not initiated. No secondary cases have occurred to our knowledge. 
In response to a large number of queries from the public and the media, the local health department established a public telephone hotline on 21 October. In addition, a third press release was issued by the Berlin health authorities on 22 October, which again informed about symptoms and transmission of meningococcal disease, and reported that close contacts of the patient during her visits to the club had contacted the health department and had been advised to obtain chemoprophylaxis. The young woman travelling to Berlin in the same compartment as the patient also contacted the health department and received chemoprophylaxis.

\section{Discussion}

This incident illustrates that close cooperation between German and Swiss health authorities led to rapid identification of potential close contacts. However, as the case was notified on a Sunday, contact tracing in Berlin was delayed until Monday morning and informing potential close contacts that had already left the hostel was not possible until 21 October, three days after the last potential contact with the infectious patient. Perhaps because of the excellent communication between the health departments in Berlin and Basel, and because of the reporting via EWRS, German authorities neglected to directly inform the national health authority in Switzerland, which does not have access to EWRS. In addition, neither the German nor the Swiss authorities informed each other in advance of the press releases they issued. Therefore, this event shows that there is still room for improvement concerning the international information exchange. More efficient communication within the European region could be achieved if Switzerland was to participate in the EWRS.

Moreover, this case illustrates the difficulty of deciding which persons require chemoprophylaxis. It is well established that short-term treatment with rifampicin, ciprofloxacin or ceftriaxone eradicates carriage of meningococci in the nasopharynx [1] and lowers the risk of secondary cases in household contacts [2]. Evidence that chemoprophylaxis lowers the risk of secondary cases in other settings is less strong, but it is generally agreed that contact with nasopharyngeal secretions, as might occur during kissing or after close contact in a confined space (such as during travel) warrants chemoprophylaxis, although there is heterogeneity in the definition of close contacts, as well as in duration and proximity criteria for fellow travellers/contacts of cases, among European countries [3]. In this case, chemoprophylaxis was definitely considered to be warranted in fellow classmates and persons who had had close contact with the patient in a club and during an overnight trip in the same train compartment, as the latter had taken place less than seven days prior to symptom onset. Assessment was more difficult with regard to the other inhabitants of the hostel, although close contact was unlikely to have occurred by having breakfast in the same room or even at the same table. It was left to the discretion of the responsible health authorities to assess whether close contact with the patient might, nonetheless, have occurred or could be ruled out. It turned out that all nine students and three supervisors of one of the German travel groups obtained chemoprophylaxis in Berlin on their own initiative prior to their departure on 22 October; while local health authorities decided that chemoprophylaxis was not necessary for members of the remaining two travel groups from Germany or the Dutch group. No secondary cases occurred in these groups as documented by follow-up as of 31 October.

\section{Conclusion}

In summary, this occurrence of fatal invasive meningococcal disease in a Swiss student while visiting Berlin highlights the importance of early disease notification. In addition, all levels of the public health system should be accessible and ready to respond at all times. Excellent communication between local health departments in Berlin and Basel led to successful identification of close contacts across European borders. When an infectious disease requiring public health action occurs in an international context, it is vital that relevant information is communicated to all levels of the public health systems of the countries involved.

\section{References}

1. Fraser A, Gafter-Gvili A, Paul M, Leibovici L. Antibiotics for preventing meningococcal infections (Review). The Cochrane Library 2005, 2005:1-35.

2. Purcell B, Samuelsson S, Hahne SJM, Ehrhard I, Heuberger S, Camaroni I, Charlett A, Stuart JM. Effectiveness of antibiotics in preventing meningococcal disease after a case: systematic review. BMJ. 2004;328(7452):1339

3. Hoek M, Hanquet G, Heuberger S, Stefanoff P, Zucs P, Ramsay M, Stuart J, on behalf of the European Union Invasive Bacterial Infections Surveillance Network (EU-IBIS). A European survey on public health policies for managing cases of meningococcal disease and their contacts. Euro Surveill. 2008;13(10):pii=8060. Available from: http://www.eurosurveillance.org/ ViewArticle.aspx?ArticleId $=8060$

This article was published on 6 November 2008

Citation style for this article: Zuschneid I, Witschi A, Quaback L, Hellenbrand W, Kleinkauf N, Koch D, Krause G. Invasive meningococcal disease with fatal outcome in a Swiss student visiting Berlin. Euro Surveill. 2008;13(45):pii=19031. Available online: http://www.eurosurveillance.org/ViewArticle.aspx?ArticleId=19031 